\title{
IDENTIDADE DE MODELOS EM FUNÇÕES DE AFILAMENTO PARA Mimosa scabrella Bentham EM POVOAMENTOS NATIVOS DA REGIÃO METROPOLITANA DE CURITIBA/PR
}

\author{
Daniel Queiroz*, Sebastião do Amaral Machado**, Afonso Figueiredo Filho***, \\ Júlio Eduardo Arce***, Henrique Soares Koehler**** \\ *Eng. Florestal, M.Sc.- valoracao@bol.com.br \\ ** Eng. Florestal, Ph.D., Depto. de Ciências Florestais, UFPR, Pesquisador 1A do CNPq - samachado@ufpr.br \\ ***Eng. Florestal, Dr., Depto. de Ciências Florestais, UFPR - afonso.figueiredo@pq.cnpq.br - jarce@ufpr.br \\ ****Eng. Florestal, Dr., Depto. de Fitotecnia e Fitossanitarismo, UFPR - koehler@ufpr.br \\ Recebido para publicação: 22/06/2007 - Aceito para publicação: 19/09/2007
}

\begin{abstract}
Resumo
Os objetivos desta pesquisa foram: avaliar a acuracidade de três funções de afilamento para estimar volumes ao longo do fuste de Mimosa scabrella por classe de idade e para o conjunto total dos dados e testar a possibilidade de agrupamento dos modelos pelo teste de identidade. A base de dados foi composta por 121 árvores cubadas pelo método de Hohenadl com dez seções, obtidas na Região Metropolitana de Curitiba (PR). As 121 árvores foram divididas em 4 classes de idade. Procedeu-se ao ajuste de três modelos para cada classe de idade, e para o conjunto total dos dados. Os modelos testados foram o de Schöepfer (1966), o de Hradetzky (1976) e o de Kozak; Munro; Smith (1969). Analisando-se os resultados, concluiu-se que o modelo de Hradetzky (1976) foi o mais adequado para estimar os volumes, em quase todas as classes de idade, e o melhor para o conjunto total dos dados. $\mathrm{O}$ teste de identidade de modelos mostrou que se pode ajustar um único modelo para as classes de idade 13 a 15 e 16 a 19 anos. As classes 6 a 9 e 10 a 12 anos somente podem ser agrupadas para os modelos Polinomial de $5^{\circ}$ Grau e para o de Kozak et al. (1969).

Palavras-chave: Funções de afilamento; teste de identidade; bracatinga; perfil do tronco.
\end{abstract}

\begin{abstract}
Identity of models in taper functions for Mimosa scabrella Bentham in native stands in the Curitiba metropolitan region. The objective of this research was: to evaluate the acuracy of three taper functions to estimate volumes along the stem of Mimosa scabrella, by age class, and by the total of the data; to test the possibility of grouping the models by the identity of models test. The data base came group 121 trees of Mymosa scabrella cubed by the method of Hohenadl with ten sections. All sample trees came from the Metropolitan Region of Curitiba (PR). The 121 trees were divided into 4 age classes. It was proceeded the adjustment of three non-segmented taper models by age class and by the total group of data. The tested models were those proposed by Schöepfer (1966), Hradetzky (1976) and Kozak; Munro; Smith (1969). After the analysis of results it was concluded that the model from Hradetzky (1976) was the best, as for estimating diameters, as for estimating volumes, for almost all age classes. It was also the best for the total data set. The identity test for the three tested models showed that it can be grouped the age classes 13 to 15 and 16-19 years. The age classes 6 to 9 and 10 to 12 only can be grouped for the $5^{\circ}$ degree and for the Kozak models.

Keywords: Taper functions; identity test; bracatinga; stem profile.
\end{abstract}

\section{INTRODUÇÃO}

O emprego de funções de afilamento no setor florestal tem proporcionado uma otimização dos recursos florestais. Essas funções, devido à sua flexibilidade, possibilitam uma estratificação, ou seja, quantificação dos múltiplos produtos existentes através de dimensões preestabelecidas.

Diversos são os fatores que influenciam na forma da árvore. Dentre eles se destacam o sítio, o comprimento e a largura de copa, a idade e a posição sociológica, dificultando a maneira certa de poder se 
estratificar e proceder aos ajustes dessas funções de afilamento. Uma maneira de testar a existência de diferença estatística entre esses fatores é através do teste de identidade.

$\mathrm{Na}$ literatura florestal brasileira, são escassos os trabalhos que aplicam o teste de identidade. Dentre os testes conhecidos, destaca-se o descrito por Graybill (1976), no qual a redução da soma de quadrado da regressão possibilita aceitar ou rejeitar uma determinada hipótese. Uma das características principais desse teste é permitir a avaliação do comportamento de uma ou mais variáveis em que se tenha interesse entre diferentes espécies. Outro caso que justifica o uso desse teste é a construção de curva de sítio em um empreendimento florestal que utiliza vários clones de uma dada espécie.

O presente trabalho teve como objetivos ajustar e comparar diferentes funções de afilamento para o total das árvores amostradas e por classe de idade, verificar a acuracidade dos modelos polinomiais não-segmentados para estimar volumes comerciais e totais ao longo do fuste, e testar a possibilidade de agrupar os modelos nas diferentes classes de idade através do teste de identidade.

\section{MATERIAL E MÉTODOS}

\section{Localização e caracterização da área de estudo}

Para o desenvolvimento desta pesquisa, foram utilizados dados de povoamentos de bracatinga localizados na região Metropolitana de Curitiba (Paraná), que inclui 14 municípios, abrangendo uma área de 876.300 ha. Esses dados foram, na sua maioria, coletados na porção norte da região, em que há uma maior concentração da espécie, incluindo os municípios de Almirante Tamandaré, Bocaiúva do Sul, Campina Grande do Sul, Campo Magro, Colombo, Itaperuçu, Rio Branco e Tunas. Esses municípios se situam entre as latitudes $25^{\circ} 11^{\prime}$ e $25^{\circ} 49^{\prime}$ S e entre as longitudes $49^{\circ} 05^{\prime}$ e $49^{\circ} 43^{\prime} \mathrm{W}$.

Segundo o sistema de classificação de Köppen, o clima da região é do tipo $\mathrm{Cfb}$, que corresponde ao clima temperado propriamente dito, mesotérmico úmido sem estação seca definida, com quatro estações bem definidas, verões quentes e chuvosos e invernos frios com períodos secos eventuais. A temperatura média do mês mais frio é inferior a $18{ }^{\circ} \mathrm{C}$, e a do mês mais quente fica abaixo de $22^{\circ} \mathrm{C}$. A temperatura média anual se apresenta em torno de $17{ }^{\circ} \mathrm{C}$, com mínimas de $12{ }^{\circ} \mathrm{C}$ e máximas de $23{ }^{\circ} \mathrm{C}$. A precipitação média anual situa-se entre 1.300 e $2.500 \mathrm{~mm}$, com leve diminuição no inverno, não ocorrendo déficits hídricos (MAACK, 1981; IAPAR, 1994).

\section{Volumetria}

Foram cubadas 121 árvores de Mimosa scabrella Bentham com idades variando de 6 a 19 anos. A cubagem dessas árvores foi feita pelo método relativo, tomando-se as medidas relativas dos diâmetros a $0 \%, 5 \%, 15 \%, 25 \%, 35 \%, 45 \%, 55 \%, 65 \%, 75 \%, 85 \%, 95 \%$ e $100 \%$ da altura total do fuste, não considerando a altura do toco. Para definir a altura de cada fuste, foi considerada a inversão morfológica do tronco mais característica, não sendo estabelecido um diâmetro mínimo.

O cálculo dos volumes das seções foi feito utilizando-se a fórmula de Hohenadl:

Sendo:

$$
\mathrm{v}=\frac{\mathrm{hf}}{10}\left[\mathrm{~g}_{0,05^{+}} \mathrm{g}_{0,15^{+}} \mathrm{g}_{0,25^{+}} \mathrm{g}_{0,35^{+}} \mathrm{g}_{0,45^{+}} \mathrm{g}_{0,55^{+}} \mathrm{g}_{0,65^{+}} \mathrm{g}_{0,75^{+}} \mathrm{g}_{0,85^{+}} \mathrm{g}_{0,95}\right]
$$

$\mathrm{v}=$ volume da árvore considerada $\left(\mathrm{m}^{3}\right)$.

$\mathrm{hf}=$ altura do fuste $(\mathrm{m})$.

$\mathrm{g}_{0, \mathrm{i}}=$ área seccional com diâmetros tomados a 5\%, 15\%, 25\%, 35\%, 45\%, 55\%, 65\%, 75\%, 85\% e $95 \%$ da altura do fuste da árvore $\left(\mathrm{m}^{2}\right)$.

As 121 árvores cubadas pelo método de Hohenadl foram distribuídas em 4 classes de idade com o mínimo de 16 árvores em cada classe de idade, conforme mostrado na tabela 1.

Tabela 1. Distribuição das árvores cubadas nas diferentes classes de idades.

Table 1. Distribution of the cubed trees in the different age classes.

\begin{tabular}{lcc}
\hline Número da classe & Classe idade (anos) & Freqüência \\
\hline 1 & $6-9$ & 24 \\
2 & $10-12$ & 55 \\
3 & $13-15$ & 26 \\
4 & $16-19$ & 16 \\
\hline Total & - & 121 \\
\hline
\end{tabular}


Os ajustes dos modelos foram feitos considerando os diâmetros a $1,3 \mathrm{~m}$ do solo (DAP) com casca, relacionados aos diâmetros comerciais com casca e as alturas relativas: $0 \%, 5 \%, 15 \%, 25 \%, 35 \%$, $45 \%, 55 \%, 65 \%, 75 \%, 85 \%, 95 \%$ e $100 \%$ da altura do fuste da árvore.

\section{Funções de afilamento não-segmentadas testadas}

$\mathrm{O}$ ajuste dos modelos não-segmentados foi feito para cada classe de idade e também para o conjunto total dos dados (não agrupados).

Os modelos não-segmentados testados são descritos a seguir:

a) Polinômio de Quinto Grau (SCHÖEPFER, 1966) - Modelo 1

Sendo:

$$
\frac{\mathrm{di}}{\mathrm{d}_{1,3}}=\beta_{0}+\beta_{1}\left(\frac{\mathrm{h}_{\mathrm{i}}}{\mathrm{hf}}\right)+\beta_{2}\left(\frac{\mathrm{h}_{\mathrm{i}}}{\mathrm{hf}}\right)^{2}+\beta_{3}\left(\frac{\mathrm{h}_{\mathrm{i}}}{\mathrm{hf}}\right)^{3}+\beta_{4}\left(\frac{\mathrm{h}_{\mathrm{i}}}{\mathrm{hf}}\right)^{4}+\beta_{5}\left(\frac{\mathrm{h}_{\mathrm{i}}}{\mathrm{hf}}\right)^{5}+\mathrm{e}_{\mathrm{i}}
$$

$\beta_{\mathrm{i}}=$ parâmetros a serem estimados.

$\mathrm{d}_{\mathrm{i}}=$ diâmetro tomado em diferentes alturas $\mathrm{h}_{\mathrm{i}}$ ao longo do fuste.

$\mathrm{d}_{1,3}=$ diâmetro a $1,3 \mathrm{~m}$ de altura (DAP).

$\mathrm{hf}=$ altura do fuste $(\mathrm{m})$.

$\mathrm{h}_{\mathrm{i}}=$ altura $\mathrm{i}(\mathrm{m})$ de um referido diâmetro.

$\mathrm{e}_{\mathrm{i}}=$ erro de estimativa.

b) Polinômio de Potências Fracionárias (HRADETZKY, 1976) - Modelo 2

Esse modelo se diferencia do polinômio de Quinto Grau por apresentar um conjunto de potências fracionárias como tentativa de melhor representar o afilamento da árvore, principalmente nos dois extremos.

Sendo:

$$
\frac{\mathrm{di}}{\mathrm{d}_{1,3}}=\beta_{0}+\beta_{1}\left(\frac{\mathrm{h}_{\mathrm{i}}}{\mathrm{hf}}\right)+\beta_{2}\left(\frac{\mathrm{h}_{\mathrm{i}}}{\mathrm{hf}}\right)^{2}+\ldots+\beta_{5}\left(\frac{\mathrm{h}_{\mathrm{i}}}{\mathrm{hf}}\right)^{\mathrm{p}_{\mathrm{n}}}+\mathrm{e}_{\mathrm{i}}
$$

$\mathrm{d}_{\mathrm{i}}, \mathrm{d}_{1,3}, \mathrm{~h}_{\mathrm{i}}$, hf, $\beta_{\mathrm{i}}$ e e $\mathrm{e}_{\mathrm{i}}=$ já definidos anteriormente.

$\mathrm{p}_{\mathrm{i}}=$ expoentes variando entre 0,00005 e 95 .

c) Polinômio de Segundo Grau (KOZAK et al., 1969) - Modelo 3

A fórmula geral do polinômio de Segundo Grau é:

$$
\frac{\mathrm{d}_{\mathrm{i}}}{\mathrm{d}_{1,3}}=\sqrt{\beta_{0}+\beta_{1}\left(\frac{\mathrm{h}_{\mathrm{i}}}{\mathrm{hf}}\right)+\beta_{2}\left(\frac{\mathrm{h}_{\mathrm{i}}}{\mathrm{hf}}\right)^{2}+\mathrm{e}_{\mathrm{i}}}
$$

Sendo:

$\mathrm{d}_{\mathrm{i}}, \mathrm{d}_{1,3}, \mathrm{~h}_{\mathrm{i}}, \mathrm{hf}, \beta_{\mathrm{i}}$ e $\mathrm{e}_{\mathrm{i}}=$ já definidos anteriormente.

Integrando esses modelos, obtém-se a expressão que propicia obter os sortimentos ou volumes comerciais correspondentes a qualquer porção da árvore, além do volume total.

\section{Acuracidade dos modelos de afilamento}

Para testar a acuracidade dos três modelos não-segmentados, foram utilizados os seguintes critérios: Análise Gráfica dos Resíduos, Coeficiente de Determinação Corrigido $\left(\mathrm{R}^{2}\right)$, Erro Padrão da Estimativa (Syx\%) e Análise Gráfica dos Perfis Médios. Esses critérios foram utilizados apenas para se verificar se os modelos possuem ajustes satisfatórios de maneira geral. Complementando o teste de acuracidade dos modelos, foram aplicadas estatísticas utilizadas por Figueiredo Filho et al. (1996), Ferreira (1999), Assis (2000), Ferreira (2004), Queiroz et al. (2006). Tais estatísticas permitem uma análise mais detalhada do desempenho das estimativas ao longo de todo o fuste, uma vez que serão calculadas para cada altura relativa em que foram tomados os diâmetros por ocasião da cubagem rigorosa. 
Foram avaliadas as variáveis volume total e volume parcial correspondente ao volume calculado a partir do método de Hohenadl. A tabela 2 apresenta as estatísticas que foram utilizadas no teste da acuracidade.

Tabela 2. Estatísticas utilizadas para avaliação dos volumes comerciais.

Table 2. Utilized statistics for evaluation of commercial volumes.

\begin{tabular}{ll}
\hline Estatísticas & Volumes \\
\hline 1) Desvio (D) & $\left(\sum\right.$ Diff $\left._{\mathrm{i}}\right) / \mathrm{N}$ \\
2) Desvio Padrão das Diferenças (SD) & {$\left[\sum\left(\text { Diff }-\mathrm{v}_{\mathrm{i}}\right)^{2} /(\mathrm{N}-1)\right]^{0,5}$} \\
$\sum\left(\text { Diff }_{\mathrm{i}} / \mathrm{v}_{\mathrm{i}}\right)^{2}$ \\
3) Soma do Quadrado do Resíduo Relativo (SQRR) \\
$\sum\left(\right.$ Diff $\left._{\mathrm{i}} / \mathrm{v}_{\mathrm{i}}\right) * 100 / \mathrm{N}$ \\
\hline
\end{tabular}

Sendo: Diff $_{\mathrm{i}}=\left(\mathrm{v}_{\mathrm{i}}-\hat{\mathrm{v}} \mathrm{i}\right)$ desvio dos volumes.

$\mathrm{v}_{\mathrm{i}}=$ volume total ou comercial até o diâmetro $\mathrm{i}$ da árvore.

$\hat{\mathrm{v}} \mathbf{i}=$ volume total ou comercial estimado.

$\mathrm{N}=$ número de árvores.

A partir dessas estatísticas, foi elaborado um ranking para expressar o desempenho dos modelos testados para estimar os volumes totais e parciais das árvores em estudo. Esse ranking foi feito para cada classe de idade e também para o conjunto total dos dados, para a variável volume. Considerando cada uma das estatísticas testadas, conforme a tabela 2, foi atribuída nota 1 para o modelo que apresentou melhor acuracidade em cada posição relativa e medição, e assim sucessivamente, até a nota 3 para a equação que propiciou as estatísticas menos acuradas. Equações com o mesmo valor das estatísticas receberam as mesmas notas.

\section{Teste de identidade}

Após a seleção da melhor equação de afilamento para cada classe de idade e para o conjunto total dos dados, foi empregado o teste de identidade, na tentativa de agrupar as classes de idade e assim possibilitar o emprego de um número menor de modelos.

Adotou-se o teste de identidade de modelos proposto por Graybill (1976). Esse teste consiste, basicamente, na redução da soma dos quadrados, permitindo verificar estatisticamente, pelo teste de F, a significância da diferença entre o total das somas dos quadrados das regressões ajustadas para cada classe de idade isoladamente (modelo completo), e a soma do quadrado da regressão ajustada para o conjunto total dos dados (modelo reduzido). Exemplificando, para o cálculo da soma de quadrados da regressão do modelo reduzido para a combinação das classes de idade 1,2, 3 e 4, faz-se o ajuste do modelo para as quatro classes juntas, e, para o cálculo da soma de quadrados da regressão do modelo completo, primeiro calcula-se individualmente para cada classe e depois faz-se o somatório.

A tabela 3 mostra o procedimento para se proceder ao cálculo da identidade dos modelos testados.

Tabela 3. Análise de variância para o teste de identidade dos modelos de afilamento.

Table 3. Analyses of variance for identity test of the taper models.

\begin{tabular}{|c|c|c|c|c|}
\hline Fonte de variação & GL & SQ & QM & $\mathbf{F}$ \\
\hline Modelo completo & $\left(C^{*} p\right)$ & $\mathrm{SQ}_{\text {(completo) }}$ & & \\
\hline Modelo reduzido & $\mathrm{P}$ & $\mathrm{SQ}_{\text {(reduzido) }}$ & & \\
\hline $\begin{array}{l}\text { Diferença para testar } \\
\text { hipótese }\end{array}$ & $(\mathrm{C}-1) \mathrm{p}$ & $S Q_{\text {(completo) }}-\mathrm{SQ}_{\text {(reduzido) }}$ & $S Q_{(\text {diferença) }} /(C-1) p$ & $\begin{array}{l}\mathrm{QM}_{\text {(diferença) }} \\
\mathrm{QM}_{\text {(reduzido) }}\end{array}$ \\
\hline Resíduos & $\mathrm{N}-\left(\mathrm{C}_{\mathrm{x}} \mathrm{p}\right)$ & $\mathrm{SQ}_{(\text {Total) }}-\mathrm{SQ}_{\text {(completo) }}$ & $\mathrm{SQ}_{\text {(reduzido) }} / \mathrm{N}-\left(\mathrm{C}_{\mathrm{x}} \mathrm{p}\right)$ & \\
\hline Total & $\mathrm{N}$ & SQ Total & - & - \\
\hline
\end{tabular}


As hipóteses testadas foram:

- $\mathrm{H} 0$ = o modelo reduzido ajustado para o conjunto total dos dados das quatro classes de idade não apresenta diferença em relação aos modelos completos ajustados para cada uma das classes pertencentes.

- $\mathrm{H} 1$ = rejeita-se $\mathrm{H} 0$.

A combinação das quatro classes de idade possibilitando a combinação de 11 hipóteses é mostrada na tabela 4 .

Tabela 4. Hipóteses de identidade para o modelo selecionado para cada classe de idade, considerando todas as combinações possíveis.

Table 4. Hypothesis of identity for the selected model for each age class considering all possible combinations

\begin{tabular}{lc}
\hline Hipóteses & Classes de Idade \\
\hline 1 & $1,2,3$ e 4 \\
2 & 1,2 e 3 \\
3 & 1,2 e 4 \\
4 & 1,3 e 4 \\
5 & 2,3 e 4 \\
6 & 1 e 2 \\
7 & 1 e 3 \\
8 & 1 e 4 \\
9 & 2 e 3 \\
10 & 2 e 4 \\
11 & 3 e 4 \\
\hline Classe $1=6$ a 9 anos; classe $2=10$ a 12 anos; classe $3=13$ a 15 anos; classe $4=16$ a 19 anos.
\end{tabular}

\section{Processamento dos dados}

Para o ajuste dos modelos de afilamento, foi utilizado o programa Statgraphics plus 2.1, visando à obtenção dos coeficientes de regressão, das estatísticas de comparação e análise de resíduos. Para o ajuste do modelo de Potências Fracionárias (HRADETZKY, 1976), utilizou-se o método Backward, o qual consiste em eliminar todas as variáveis que não atingem o grau de significância determinado para o teste. Houve uma combinação de potências que foi desde 0,00005 até 95 .

As informações obtidas através desse processamento foram organizadas, a fim de se realizarem as análises estatísticas.

\section{RESULTADOS E DISCUSSÃO}

\section{Ajustes dos modelos}

Os parâmetros estimados para os três modelos testados por classe de idade e para o conjunto total dos dados são apresentados na tabela 5, com os respectivos Coeficientes de Determinação Corrigidos $\left(\mathrm{R}^{2}\right)$ e Erros Padrões da Estimativa em percentagem (Syx\%). Essas estatísticas retratam valores médios, não fornecendo assim nenhum indicativo do comportamento ao longo do fuste. Esses testes foram complementados pelas estatísticas constantes da tabela 2, permitindo assim uma análise mais detalhada.

Em geral, os modelos testados apresentaram Coeficiente de Determinação $\left(\mathrm{R}^{2}\right)$ inferior a 0,90 e Erro Padrão da Estimativa (Syx\%) entre 10 e 15\%. Apenas na classe de idade 6 a 9 anos e para o conjunto total de árvores o $\mathrm{R}^{2}$ foi maior que 0,90 para todos os modelos testados. A equação de Kozak et al. (1969) mostrou os menores valores de $\mathrm{R}^{2}$ em todas as classes de idade, quando comparados com os valores dos outros dois modelos testados.

\section{Acuracidade dos modelos não-segmentados na estimativa dos volumes}

A figura 1 mostra a análise gráfica dos resíduos para o conjunto total dos dados (não agrupados). Esses gráficos consistiram em analisar os resíduos percentuais $(\%)$ em função do volume estimado $\left(\mathrm{m}^{3}\right)$, sendo elaborados para cada classe de idade. Observou-se tendenciosidade na estimativa dos diâmetros em todas classes de idade. Nem sempre a equação de afilamento que apresentou a melhor pontuação, conforme será mostrado a seguir, deixa de apresentar tendenciosidade quando usada para estimativa do 
volume ao longo da linha média, indicando que a pontuação não pode ser usada exclusivamente na escolha de um modelo.

Como mostrado na figura 1 , em geral todos os modelos apresentaram tendenciosidade ao longo da linha estimativa. Os três modelos testados para as diferentes classes de idade apresentaram alguma tendenciosidade ao longo da linha média.

Tabela 5. Parâmetros estimados e respectivas medidas de precisão, para as quatro classes de idade e para os modelos ajustados por classe de idade e para o conjunto total dos dados.

Table 5. Estimated parameters and mensures of precision for the fitted models by age classes and for the whole data set.

\begin{tabular}{|c|c|c|c|c|c|c|c|c|}
\hline \multirow{3}{*}{$\begin{array}{l}\text { Classe idade } \\
\text { (anos) }\end{array}$} & \multicolumn{8}{|c|}{ Modelo do Quinto Grau - Modelo 1} \\
\hline & \multicolumn{8}{|c|}{ Parâmetros estimados } \\
\hline & $\hat{\beta}_{0}$ & $\hat{\beta}_{1}$ & $\hat{\beta}_{2}$ & $\hat{\beta}_{3}$ & $\hat{\beta}_{4}$ & $\hat{\beta}_{5}$ & $\mathrm{R}^{2}$ & Syx $\%(\hat{d} \mathrm{i})$ \\
\hline $6-9$ & $1,18136^{* *}$ & $-2,1841 * *$ & $7,20916^{* *}$ & $-13,2895 * *$ & $11,213^{* *}$ & $-3,56757 * *$ & 0,93901 & 10,7046 \\
\hline $10-12$ & $1,25787 * *$ & $-3,74865 * *$ & $16,2077 * *$ & $-33,3579 * *$ & $30,388 * *$ & $-10,1582 * *$ & 0,90496 & 11,7342 \\
\hline $13-15$ & $1,19409 * *$ & $-2,41612 * *$ & $8,75301 * *$ & $-16,4941 * *$ & $14,188^{* *}$ & $-4,54168 * *$ & 0,89270 & 11,5992 \\
\hline $16-19$ & $1,20971 * *$ & $-2,75841 * *$ & $10,4931 * *$ & $-21,0139 * *$ & $19,187 * *$ & $-6,42512 * *$ & 0,76225 & 11,9915 \\
\hline Total & $1,22262 * *$ & $-3,02105 * *$ & $12,0654 * *$ & $-24,1215 * *$ & $21,622 * *$ & $-7,15046^{* *}$ & 0,92437 & 12,6160 \\
\hline
\end{tabular}

\begin{tabular}{|c|c|c|c|c|c|c|c|}
\hline \multirow{3}{*}{$\begin{array}{l}\text { Classe idade } \\
\text { (anos) }\end{array}$} & \multicolumn{7}{|c|}{ Modelo de Potências Fracionárias - Modelo 2} \\
\hline & \multicolumn{7}{|c|}{ Parâmetros estimados } \\
\hline & $\hat{\beta}_{0}$ & $\hat{\beta}_{1}$ & $\hat{\beta}_{2}$ & $\hat{\beta}_{3}$ & $\hat{\beta}_{4}$ & $\mathrm{R}^{2}$ & $\operatorname{Syx} \%(\hat{d i})$ \\
\hline $\begin{array}{l}6-9 \\
\text { Potências }\end{array}$ & $1,1913 * *$ & $\begin{array}{c}-0,067119 * * \\
0,00005\end{array}$ & $\begin{array}{c}-0,50608 * * \\
0,7\end{array}$ & $\begin{array}{c}-0,057774 * * \\
5\end{array}$ & & 0,93966 & 10,6474 \\
\hline $\begin{array}{l}10-12 \\
\text { Potências }\end{array}$ & $1,28259 * *$ & $\begin{array}{c}-0,125363^{* *} \\
0,00005 \\
\end{array}$ & $\begin{array}{c}-0,438911^{* *} \\
0,5 \\
\end{array}$ & $\begin{array}{c}-0,212281^{* *} \\
4 \\
\end{array}$ & $\begin{array}{c}0,0958567^{* *} \\
18 \\
\end{array}$ & 0,90864 & 11,5051 \\
\hline $\begin{array}{l}13-15 \\
\text { Potências } \\
\end{array}$ & $1,19665 * *$ & $\begin{array}{c}-0,505281^{* *} \\
0,5 \\
\end{array}$ & & & & 0,89470 & 11,4904 \\
\hline $\begin{array}{l}16-19 \\
\text { Potências }\end{array}$ & $1,23251 * *$ & $\begin{array}{c}-0,549377^{* *} \\
0,4 \\
\end{array}$ & & & & 0,76648 & 11,8844 \\
\hline $\begin{array}{l}\text { Total } \\
\text { Potências }\end{array}$ & $1,24109^{* *}$ & $\begin{array}{c}-0,07589^{* *} \\
0,00005\end{array}$ & $\begin{array}{c}-0,459412 * * \\
0,5 \\
\end{array}$ & $\begin{array}{c}-0,145145^{* *} \\
4 \\
\end{array}$ & $\begin{array}{c}0,0654363^{* *} \\
17\end{array}$ & 0,92603 & 12,4767 \\
\hline
\end{tabular}

\begin{tabular}{lccccc} 
Potências & 0,00005 & 0,5 & 4 & 17 & \\
\hline \multicolumn{5}{c}{ Modelo de Kozak et al. (1969) - Modelo 3 } \\
\hline Classe idade & \multicolumn{5}{c}{ Parâmetros estimados } \\
\cline { 2 - 6 } (anos) & $\hat{\beta}_{0}$ & $\hat{\beta}_{1}$ & $\hat{\beta}_{2}$ & $\mathrm{R}^{2}$ & Syx\% $(\hat{\mathrm{d}} \mathbf{i})$ \\
\hline $6-9$ & $1,29196^{* *}$ & $-1,67533^{* *}$ & $0,743909^{* *}$ & 0,93072 & 11,4091 \\
$10-12$ & $1,39729^{* *}$ & $-2,00489^{* *}$ & $1,01853^{* *}$ & 0,88370 & 12,9807 \\
$13-15$ & $1,31091^{* *}$ & $-1,66001^{* *}$ & $0,869279^{* *}$ & 0,88048 & 12,2420 \\
$16-19$ & $1,3409^{* *}$ & $-1,99327^{* *}$ & $1,19017^{* *}$ & 0,74492 & 12,4208 \\
Total & $1,35038^{* *}$ & $-1,86388^{* *}$ & $0,954686^{* *}$ & 0,91545 & 13,3395 \\
\hline
\end{tabular}

** Significativo ao nível de probabilidade de $95 \%$.

A tabela 6 apresenta as estatísticas: Desvio (D); Soma de Quadrados dos Resíduos Relativos (SQRR), Desvio Padrão das Diferenças (SD) e Resíduo Percentual (RP). Essa tabela apresenta resultados obtidos pelas três equações polinomiais não-segmentadas para o ajuste do total dos dados (não agrupados), para a variável volume em cada altura relativa do fuste, na qual os volumes são acumulativos. Exemplificando: os volumes são calculados de 0 até $10 \%$ da altura relativa, de 0 a $20 \%$ e assim até totalizar o volume total ( 0 a $100 \%$ ). Na tabela 7 , podem-se observar os resultados para a classe de idade de 10 a 12 anos.

Em geral, os valores da estatística Desvios (D) foram próximos de zero para a maioria dos casos. Como mostrado na tabela 6, a equação de Hradetzky foi a que apresentou os menores valores no somatório na maioria das posições, principalmente nas posições inferiores do fuste (até a altura de $60 \%$ ), sendo, portanto o modelo mais acurado para a estimativa do volume para o conjunto total dos dados. 
QUIN TO GRAU

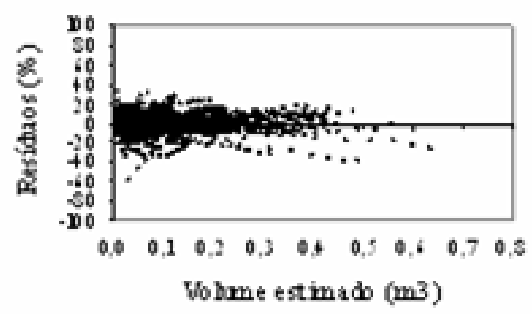

PO TENC LAS FRAC IONÁRLAS

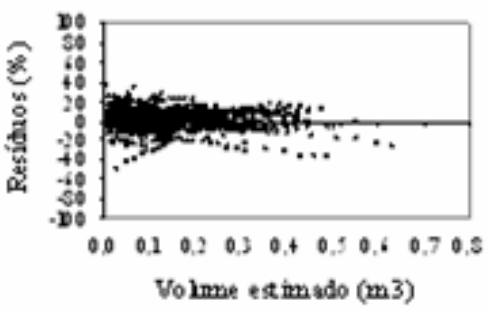

KOTAK et al. (196)

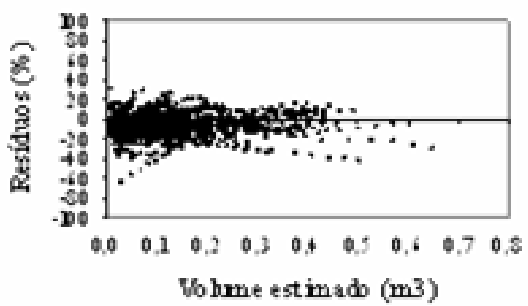

Figura 1. Distribuição gráfica dos resíduos em percentagem em função do volume estimado para o conjunto total dos dados para os três modelos ajustados.

Figure 1. Graphic residual distribution in percentage over estimated volume for the total data set for the three fitted equations.

Tabela 6. Estatísticas dos modelos polinomiais não-segmentados para a estimativa dos volumes ao longo do fuste das árvores de Mimosa scabrella, para o ajuste do conjunto total dos dados (não agrupados).

Table 6. Statistics of the fitted taper equations for volume estimate for the total data set.

\begin{tabular}{|c|c|c|c|c|c|c|c|c|c|c|c|}
\hline \multirow{2}{*}{ Modelo } & \multirow{2}{*}{ Estatística } & \multicolumn{10}{|c|}{ Alturas relativas de medição (\%) } \\
\hline & & 10 & 20 & 30 & 40 & $\mathbf{5 0}$ & 60 & 70 & 80 & 90 & 100 \\
\hline \multirow{4}{*}{$5^{\circ} \mathrm{Grau}$} & $\mathrm{D}$ & $-0,003$ & $-0,002$ & $-0,002$ & $-0,002$ & $-0,002$ & $-0,003$ & $-0,002$ & $-0,002$ & $-0,002$ & $-0,002$ \\
\hline & SQRR & 2,271 & 0,952 & 0,737 & 0,729 & 0,793 & 0,894 & 1,053 & 1,216 & 1,406 & 1,721 \\
\hline & SD & 0,040 & 0,070 & 0,098 & 0,124 & 0,148 & 0,170 & 0,189 & 0,205 & 0,220 & 0,233 \\
\hline & $\mathrm{RP}$ & $-7,048$ & $-2,602$ & $-1,107$ & $-0,802$ & $-0,840$ & $-0,848$ & $-0,458$ & 0,011 & 0,080 & 0,140 \\
\hline \multirow{4}{*}{$\begin{array}{l}\text { Hradetzky } \\
\text { (1976) }\end{array}$} & $\mathrm{D}$ & 0,000 & $-0,001$ & $-0,001$ & $-0,001$ & 0,000 & $-0,001$ & $-0,001$ & 0,000 & 0,000 & 0,000 \\
\hline & SQRR & 1,931 & 0,917 & 0,739 & 0,738 & 0,808 & 0,911 & 1,076 & 1,239 & 1,434 & 1,761 \\
\hline & SD & 0,037 & 0,069 & 0,097 & 0,123 & 0,146 & 0,168 & 0,187 & 0,204 & 0,218 & 0,231 \\
\hline & $\mathrm{RP}$ & $-0,174$ & $-0,047$ & $-0,036$ & 0,243 & 0,464 & 0,445 & 0,543 & 0,724 & 0,802 & 0,984 \\
\hline \multirow{5}{*}{$\begin{array}{l}\text { Kozak et } \\
\text { al. (1969) }\end{array}$} & $\mathrm{D}$ & $-0,003$ & $-0,007$ & $-0,009$ & $-0,009$ & $-0,008$ & $-0,008$ & $-0,006$ & $-0,005$ & $-0,005$ & $-0,006$ \\
\hline & SQRR & 2,682 & 1,943 & 1,636 & 1,334 & 1,129 & 1,058 & 1,109 & 1,219 & 1,396 & 1,701 \\
\hline & $\mathrm{SD}$ & 0,041 & 0,076 & 0,107 & 0,133 & 0,156 & 0,176 & 0,194 & 0,210 & 0,224 & 0,239 \\
\hline & RP & $-9,507$ & $-10,001$ & $-9,259$ & $-7,686$ & $-5,930$ & $-4,425$ & $-3,085$ & $-2,151$ & $-1,991$ & $-2,274$ \\
\hline & \multicolumn{11}{|c|}{ Notas } \\
\hline \multicolumn{2}{|l|}{$5^{\circ} \mathrm{Grau}$} & 8 & 8 & 8 & 8 & 7 & 7 & 6 & 6 & 7 & 7 \\
\hline \multicolumn{2}{|c|}{ Hradetzky (1976) } & 4 & 4 & 4 & 4 & 5 & 5 & 6 & 7 & 7 & 7 \\
\hline \multicolumn{2}{|c|}{ Kozak et al. (1969) } & 12 & 12 & 12 & 12 & 12 & 12 & 12 & 11 & 10 & 10 \\
\hline
\end{tabular}

$\mathrm{D}=$ desvio; $\mathrm{SQRR}=$ soma de quadrados dos resíduos relativos; $\mathrm{SD}=$ desvio padrão das diferenças; $\mathrm{R}$ = resíduo percentual. 
A partir dessas estatísticas, foi feita uma classificação das equações, de modo a permitir a escolha da equação mais acurada para estimar os volumes parciais acumulados nas diferentes posições do fuste, sendo que a equação que obteve as menores notas foi considerada a mais adequada. Quando duas equações sao classificadas em uma mesma posição, significa que apresentam o mesmo número de pontos. Quando se calculou a percentagem no caso dessas equações empatadas, considerou-se o peso de 0,5 para cada uma, de modo que o somatório totalizasse $100 \%$ (Tabela 8 ).

Tabela 7. Estatísticas dos modelos polinomiais não-segmentados para a estimativa dos volumes ao longo do fuste das árvores de Mimosa scabrella, na classe de idade 10 a 12 anos.

Table 7. Statistics of the fitted taper equations for volume estimates for the age class 10 to 12 years old.

\begin{tabular}{|c|c|c|c|c|c|c|c|c|c|c|c|}
\hline \multirow{2}{*}{ Modelo } & \multirow{2}{*}{ Estatística } & \multicolumn{10}{|c|}{ Alturas relativas de medição (\%) } \\
\hline & & 10 & 20 & 30 & 40 & 50 & 60 & 70 & 80 & 90 & 100 \\
\hline \multirow{4}{*}{$5^{\circ} \mathrm{Grau}$} & $\mathrm{D}$ & $-0,003$ & $-0,003$ & $-0,002$ & $-0,002$ & $-0,002$ & $-0,003$ & $-0,003$ & $-0,003$ & $-0,003$ & $-0,004$ \\
\hline & SQRR & 1,144 & 0,432 & 0,352 & 0,343 & 0,374 & 0,422 & 0,469 & 0,525 & 0,622 & 0,751 \\
\hline & $\mathrm{SD}$ & 0,034 & 0,059 & 0,082 & 0,104 & 0,124 & 0,142 & 0,158 & 0,171 & 0,182 & 0,192 \\
\hline & $\mathrm{RP}$ & $-10,069$ & $-3,902$ & $-2,149$ & $-1,851$ & $-1,731$ & $-1,720$ & $-1,323$ & $-0,806$ & $-0,600$ & $-0,719$ \\
\hline \multirow{4}{*}{$\begin{array}{l}\text { Hradetzky } \\
\text { (1976) }\end{array}$} & $\mathrm{D}$ & $-0,001$ & $-0,001$ & $-0,001$ & $-0,001$ & 0,000 & $-0,001$ & $-0,001$ & $-0,001$ & $-0,001$ & $-0,002$ \\
\hline & SQRR & 0,716 & 0,375 & 0,339 & 0,335 & 0,373 & 0,422 & 0,474 & 0,533 & 0,634 & 0,768 \\
\hline & $\mathrm{SD}$ & 0,031 & 0,057 & 0,081 & 0,102 & 0,122 & 0,140 & 0,155 & 0,169 & 0,180 & 0,190 \\
\hline & $\mathrm{RP}$ & $-0,775$ & $-0,464$ & $-0,603$ & $-0,232$ & 0,265 & 0,207 & 0,131 & 0,209 & 0,436 & 0,510 \\
\hline \multirow{5}{*}{$\begin{array}{l}\text { Kozak et } \\
\text { al. (1969) }\end{array}$} & $\mathrm{D}$ & $-0,005$ & $-0,008$ & $-0,010$ & $-0,010$ & $-0,009$ & $-0,008$ & $-0,007$ & $-0,007$ & $-0,007$ & $-0,009$ \\
\hline & SQRR & 1,537 & 1,243 & 1,071 & 0,826 & 0,635 & 0,557 & 0,535 & 0,557 & 0,642 & 0,775 \\
\hline & $\mathrm{SD}$ & 0,035 & 0,065 & 0,091 & 0,113 & 0,132 & 0,149 & 0,163 & 0,175 & 0,187 & 0,198 \\
\hline & $\mathrm{RP}$ & $-13,425$ & $-13,203$ & $-12,119$ & $-10,030$ & $-7,615$ & $-5,784$ & $-4,339$ & $-3,363$ & $-3,079$ & $-3,542$ \\
\hline & \multicolumn{11}{|c|}{ Notas } \\
\hline \multicolumn{2}{|l|}{$5^{\circ} \mathrm{Grau}$} & 8 & 8 & 8 & 8 & 8 & 7 & 7 & 7 & 7 & 7 \\
\hline \multicolumn{2}{|c|}{ Hradetzky (1976) } & 4 & 4 & 4 & 4 & 4 & 5 & 5 & 5 & 5 & 5 \\
\hline \multicolumn{2}{|c|}{ Kozak et al. (1969) } & 12 & 12 & 12 & 12 & 12 & 12 & 12 & 12 & 12 & 12 \\
\hline
\end{tabular}

Para o conjunto total de dados, a equação de Potências foi a que estimou os volumes com maior acuracidade, representando $75 \%$ das posições, seguida pela equação de $5^{\circ}$ Grau, com $25 \%$. Na tabela 8 , fica evidente a superioridade da equação de Potências ao longo de todo o fuste, com exceção apenas da classe de 6 a 9 anos, na qual a equação do Polinômio de $5^{\circ}$ Grau foi a que ocupou o primeiro lugar, com $90 \%$. Há um destaque para a equação de Potências na classe de 10 a 12 anos, em que representou $100 \%$ das posições.

Tabela 8. Classificação das equações nas posições do fuste por classe de idade e para o conjunto total dos dados, e valores percentuais nas ocasiões em que o modelo ocupou a primeira posição para estimativa dos volumes totais e parciais.

Table 8. Ranking of the fitted equations at the stem positions by age class and by the whole data set (total) for volume estimate at every position along the tree stem.

\begin{tabular}{lccccc}
\hline \multirow{2}{*}{ Altura (\%) } & \multicolumn{5}{c}{ Classes de idade (anos) } \\
\cline { 2 - 6 } & $\mathbf{6 - 9}$ & $\mathbf{1 0}-\mathbf{1 2}$ & $\mathbf{1 3 - 1 5}$ & $\mathbf{1 6}-\mathbf{1 9}$ & TOTAL \\
\hline 10 & $\mathrm{Q}$ & $\mathrm{H}$ & $\mathrm{H}$ & $\mathrm{H}$ & $\mathrm{H}$ \\
20 & $\mathrm{Q}$ & $\mathrm{H}$ & $\mathrm{Q}$ & $\mathrm{H}$ & $\mathrm{H}$ \\
30 & $\mathrm{Q}$ & $\mathrm{H}$ & $\mathrm{Q}$ & $\mathrm{Q}-\mathrm{H}$ & $\mathrm{H}$ \\
40 & $\mathrm{Q}$ & $\mathrm{H}$ & $\mathrm{Q}-\mathrm{H}$ & $\mathrm{H}$ \\
50 & $\mathrm{Q}$ & $\mathrm{H}$ & $\mathrm{H}$ & $\mathrm{Q}-\mathrm{H}$ & $\mathrm{H}$ \\
60 & $\mathrm{Q}$ & $\mathrm{H}$ & $\mathrm{H}$ & $\mathrm{Q}$ & $\mathrm{Q}-\mathrm{H}$ \\
70 & $\mathrm{Q}$ & $\mathrm{H}$ & $\mathrm{Q}-\mathrm{H}$ & $\mathrm{Q}-\mathrm{H}$ & $\mathrm{Q}$ \\
80 & $\mathrm{Q}$ & $\mathrm{H}$ & $\mathrm{Q}-\mathrm{H}$ & $\mathrm{Q}-\mathrm{H}$ & $\mathrm{Q}-\mathrm{H}$ \\
90 & $\mathrm{Q}-\mathrm{K}$ & $\mathrm{H}$ & $\mathrm{H}$ & $\mathrm{Q}-\mathrm{H}$ & $\mathrm{Q}-\mathrm{H}$ \\
100 & $\mathrm{Q}-\mathrm{K}$ & $100 \%$ & $50 \%$ & $40 \%$ & $25 \%$ \\
\hline $\mathrm{Q}$ & $90 \%$ & $50 \%$ & $60 \%$ & $75 \%$ \\
$\mathrm{H}$ & $0 \%$ & $0 \%$ & $0 \%$ & $0 \%$ \\
$\mathrm{~K}$ & $10 \%$ & $0 \%$ & & $00 \%$ \\
\hline
\end{tabular}

Modelos: Q= Polinômio de Quinto Grau; H= Potências Fracionárias (HRADETZKY, 1976); K= Kozak et al. (1969). 

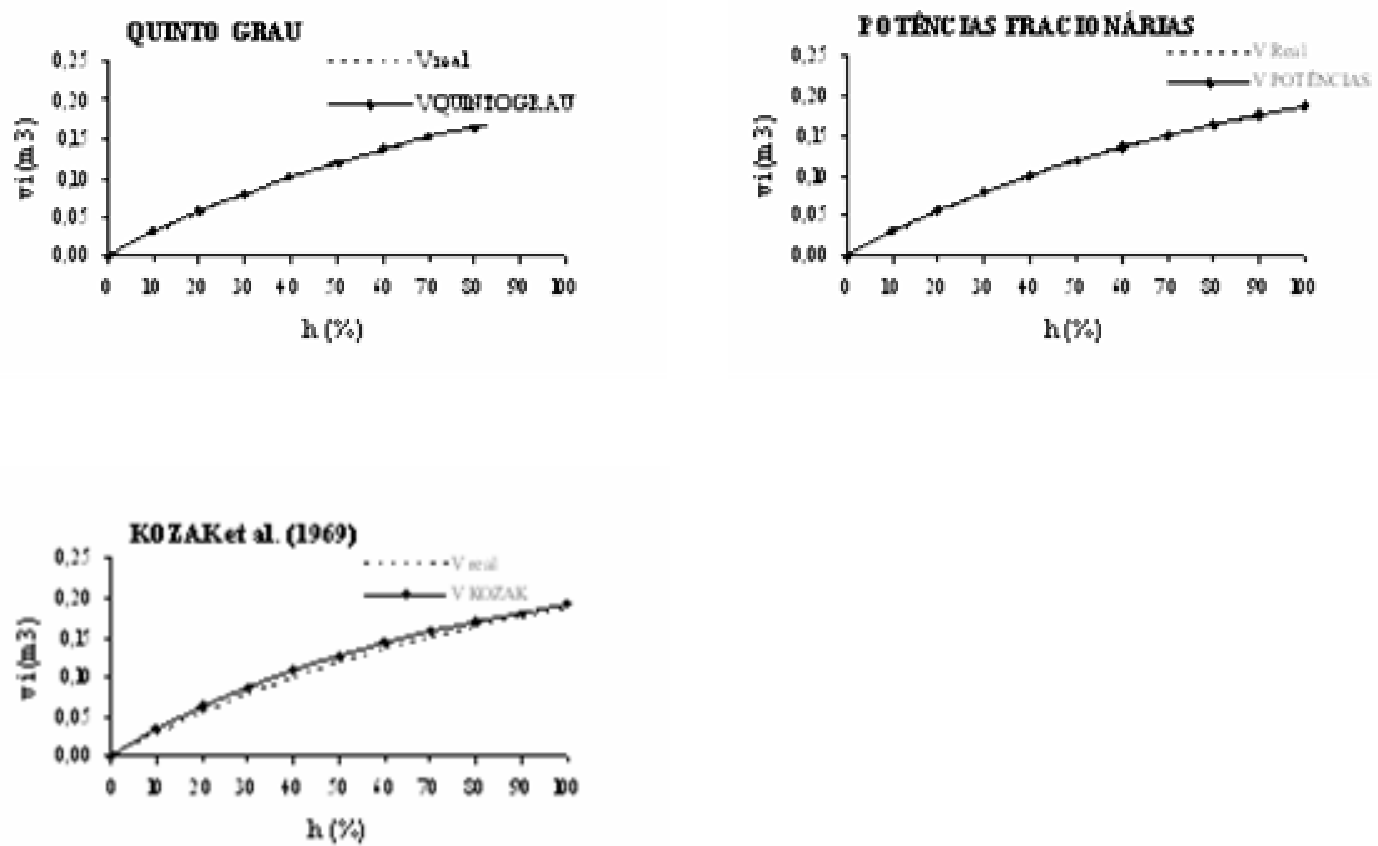

Figura 2. Volumes reais e estimados pelos modelos polinomiais não-segmentados para o conjunto total dos dados para os três modelos ajustados.

Figure 2. Observed and estimated volumes by the tree fitted taper equations for the total data set.

\section{Teste de identidade dos modelos}

Esse teste tem como objetivo o agrupamento das diferentes classes de idade, visando ajustar um único modelo para o conjunto total dos dados. Ao todo, foram feitas 11 combinações entre as 4 classes de idade. As tabelas 9, 10 e 11 mostram os resultados do teste de identidade de modelo para o Polinômio de $5^{\circ}$ Grau, o Polinômio de Potências Fracionárias e o Polinômio de Kozak, respectivamente. Essas tabelas também apresentam os quadrados médios da diferença e dos resíduos provenientes da análise de variância da regressão (ANOVA), que possibilitaram o ajuste dos polinômios. Dessa forma, sempre que o valor de

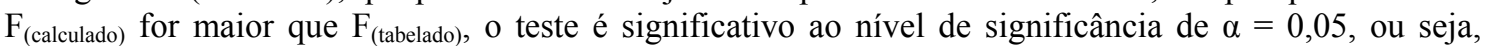
rejeita-se $\mathrm{H}_{0}$. Então, a soma de quadrados dos resíduos de cada classe de idade difere estatisticamente da soma de quadrados dos resíduos feita para o conjunto total dos dados. Quando ocorrer o contrário, o teste é não-significativo - então aceita-se $\mathrm{H}_{0}$.

A primeira combinação para o conjunto total dos dados (combinação 1, 2, 3 e 4) é apresentada nas tabelas 9, 10 e 11. Caso o teste fosse não-significativo ao nível de probabilidade de 95\%, partir-se-ia do princípio de que não precisaria estratificar por classe de idade. No entanto, como o resultado foi significativo, deu-se continuidade ao teste para as diferentes combinações.

Para todos os três modelos testados, o teste de identidade mostrou o mesmo comportamento para a combinação das classes de idade de 3 e 4, ou seja, poderá ser ajustado um único modelo (modelo reduzido).

As tabela 9 e 11 mostraram a possibilidade de agrupamento das classes de idade 1 e 2 por apresentarem $P\left(F_{\mathrm{v} 1, \mathrm{v} 2}>\mathrm{F}_{\text {calculado }}\right)=0,88619$ e 0,72392 , respectivamente.

Em geral, para as demais combinações de idades, considerando-se os três modelos testados, o teste de $\mathrm{F}$ indicou que é inadequado o agrupamento das classes de idade para a obtenção de equações reduzidas, devendo ser ajustadas equações individuais para cada classe de idade (modelo completo). 
Tabela 9. Resultado do teste de identidade das equações por meio do teste de F, para as 11 combinações de idades - Polinômio de $5^{\circ}$ Grau.

Table 9. Results from the test of identify by the $\mathrm{F}$ test for the 11 possible age combinations -5 th degree polynomial.

\begin{tabular}{|c|c|c|c|c|c|c|}
\hline Combinações & $\begin{array}{c}\text { QM } \\
\text { (diferença) }\end{array}$ & $\begin{array}{c}\mathbf{Q M} \\
\text { (resíduo) }\end{array}$ & $F_{\text {calculado }}$ & $\mathbf{F}_{\text {tabelado 5\% }}$ & $\begin{array}{c}\text { Valor }-p \\
P\left(F_{v 1, v 2}>F_{\text {calculado }}\right)\end{array}$ & Teste \\
\hline $1,2,3$ e 4 & 0,03789 & 0,00879 & 4,311 & 1,73798 & 0,99996 & $\mathrm{~S}$ \\
\hline 1,2 e 3 & 0,04116 & 0,00847 & 4,857 & 1,92193 & 0,99986 & S \\
\hline 1,2 e 4 & 0,03187 & 0,00869 & 3,665 & 1,92247 & 0,99896 & S \\
\hline 1,3 e 4 & 0,02667 & 0,00809 & 3,295 & 1,92498 & 0,99784 & S \\
\hline 2,3 e 4 & 0,04787 & 0,00969 & 4,938 & 1,92235 & 0,99987 & S \\
\hline 1 e 2 & 0,01517 & 0,00826 & 1,837 & 2,30204 & $\mathbf{0 , 8 8 6 1 9}$ & NS \\
\hline 1 e 3 & 0,03697 & 0,00721 & 5,129 & 2,30548 & 0,99864 & S \\
\hline 1 e 4 & 0,03545 & 0,00741 & 4,785 & 2,30785 & 0,99806 & $\mathrm{~S}$ \\
\hline 2 e 3 & 0,06910 & 0,00946 & 7,302 & 2,30190 & 0,99978 & $\mathrm{~S}$ \\
\hline 2 e 4 & 0,04841 & 0,00990 & 4,892 & 2,30271 & 0,99829 & S \\
\hline 3 e 4 & 0,00605 & 0,00980 & 0,617 & 2,30728 & $\mathbf{0 , 0 8 2 2 7}$ & NS \\
\hline
\end{tabular}

v1= Grau de Liberdade do denominador; v2= Grau de Liberdade do numerador; $\mathrm{S}=$ Significativo ao nível de $95 \%$ de probabilidade pela distribuição F; NS= Não-significativo ao nível de $95 \%$ de probabilidade pela distribuição F. Classe $1=6$ a 9 anos; Classe $2=10$ a 12 anos; Classe 3=13 a 15 anos; Classe 4= 16 a 19 anos.

Tabela 10. Resultado do teste de identidade das equações por meio do teste de F, para as 11 combinações de idade estudadas para o Polinômio de Potências Fracionárias.

Table 10. Result from the test of identity of equations for 11 possible age combinations - power polynomial.

\begin{tabular}{|c|c|c|c|c|c|c|}
\hline Combinações & $\mathbf{Q M}$ & $\mathbf{Q M}$ & $F_{\text {calculado }}$ & $F_{\text {tabelado } 5 \%}$ & Valor $-\mathbf{p}$ & Teste \\
\hline $1,2,3$ e 4 & 0,04326 & 0,00854 & 5,068 & 1,84775 & 0,99996 & $\mathrm{~S}$ \\
\hline 1,2 e 3 & 0,04616 & 0,00821 & 5,623 & 2,07058 & 0,99980 & $\mathrm{~S}$ \\
\hline 1,2 e 4 & 0,03985 & 0,00842 & 4,735 & 2,07108 & 0,99942 & $\mathrm{~S}$ \\
\hline 1,3 e 4 & 0,04921 & 0,00796 & 6,182 & 2,71301 & 0,99743 & $\mathrm{~S}$ \\
\hline 2,3 e 4 & 0,07341 & 0,00939 & 7,815 & 2,30094 & 0,99985 & $\mathrm{~S}$ \\
\hline 1 e 2 & 0,02190 & 0,00796 & 2,751 & 2,54335 & 0,96202 & $\mathrm{~S}$ \\
\hline 1 e 3 & 0,04731 & 0,00709 & 6,673 & 2,93561 & 0,99660 & $\mathrm{~S}$ \\
\hline $1 \mathrm{e} 4$ & 0,06998 & 0,00730 & 9,581 & 3,67800 & 0,99591 & $\mathrm{~S}$ \\
\hline 2 e 3 & 0,07400 & 0,00914 & 8,097 & 2,54320 & 0,99954 & $\mathrm{~S}$ \\
\hline 2 e 4 & 0,08641 & 0,00955 & 9,047 & 2,93322 & 0,99887 & $\mathrm{~S}$ \\
\hline 3 e 4 & $\mathbf{0 , 0 1 0 5 3}$ & 0,00962 & 1,095 & 5,63531 & 0,45595 & NS \\
\hline
\end{tabular}

\section{CONCLUSÕES}

- A equação de Potências Fracionárias é a mais acurada na estimativa dos volumes em quase todas classes de idade, e a melhor para o conjunto total dos dados.

- O modelo de Kozak et al. (1969) apresentou tendenciosidade ao longo do fuste na estimativa do volume, ora superestimando, ora subestimando em todas as classes de idade e no conjunto total dos dados, e portanto não deve ser utilizado nas estimativas.

- O modelo de Potências Fracionárias mostrou uma alta eficiência na estimativa dos diâmetros, principalmente nas partes inferiores do fuste;

- O teste de identidade para os modelos testados revelou que se podem agrupar os dados das classes de idade 13-15 e 16-19 anos. As classes 6-9 e 10-12 anos somente podem ser agrupadas para os modelos Polinômio de $5^{\circ}$ Grau e de Kozak et al. (1969). 
Tabela 11. Resultado do teste de identidade das equações por meio do teste de F, para as 11 combinações dentro das quatro classes de idade estudadas para o Polinômio de Kozak et al. (1969).

Table 11. Identity's test result from equations by the F test for 11 possible combinations into 4 age classes studied in Kozak et al Polynomial (1969).

\begin{tabular}{lcccccc}
\hline Combinações & $\begin{array}{c}\mathbf{Q M} \\
\text { (diferença) }\end{array}$ & $\begin{array}{c}\mathbf{Q M} \\
\text { (resíduo) }\end{array}$ & $\mathbf{F}_{\text {calculado }}$ & $\mathbf{F}_{\text {tabelado 5\% }}$ & $\begin{array}{c}\text { Valor }-\mathbf{p} \\
\left(\mathbf{F}_{\mathbf{v 1}, \mathbf{v} 2}>\mathbf{F}_{\text {calculado }}\right)\end{array}$ & Teste \\
\hline 1, 2, 3 e 4 & 0,15639 & 0,03776 & 4,142 & 2,30000 & 0,99618 & $\mathrm{~S}$ \\
1, 2 e 3 & 0,17178 & 0,03768 & 4,559 & 2,71065 & 0,99181 & $\mathrm{~S}$ \\
1, 2 e 4 & 0,12792 & 0,03894 & 3,285 & 2,71106 & 0,97356 & $\mathrm{~S}$ \\
1, 3 e 4 & 0,09517 & 0,02932 & 3,245 & 2,71301 & 0,97236 & $\mathrm{~S}$ \\
2, 3 e 4 & 0,20923 & 0,04241 & 4,933 & 2,71098 & 0,99390 & $\mathrm{~S}$ \\
1 e 2 & $\mathbf{0 , 0 6 4 2 3}$ & $\mathbf{0 , 0 3 9 0 7}$ & $\mathbf{1 , 6 4 4}$ & $\mathbf{3 , 6 7 3 4 6}$ & $\mathbf{0 , 7 2 3 9 2}$ & NS \\
1 e 3 & 0,11560 & 0,02647 & 4,368 & 3,67615 & 0,96726 & $\mathrm{~S}$ \\
1 e 4 & 0,13327 & 0,02664 & 5,002 & 3,67800 & 0,97673 & $\mathrm{~S}$ \\
2 e 3 & 0,31147 & 0,04321 & 7,207 & 3,67335 & 0,99114 & $\mathrm{~S}$ \\
2 e 4 & 0,19597 & 0,04569 & 4,289 & 3,67399 & 0,96580 & $\mathrm{~S}$ \\
3 e 4 & $\mathbf{0 , 0 3 4 5 3}$ & $\mathbf{0 , 0 3 5 2 8}$ & $\mathbf{0 , 9 7 9}$ & $\mathbf{3 , 6 7 7 5 6}$ & $\mathbf{0 , 4 1 0 1 6}$ & NS \\
\hline
\end{tabular}

\section{REFERÊNCIAS}

ASSIS, A. L. de. Avaliação de modelos polinomiais segmentados e não-segmentados na estimativa de diâmetros e volumes comerciais de Pinus taeda. 189 p. Dissertação (Mestrado em Engenharia Florestal) - Universidade Federal de Lavras, Lavras, 2000.

FERREIRA, M. Z. Estudo de funções de afilamento para representar o perfil e o volume do fuste de Pinus taeda L. 196 p. Dissertação (Mestrado em Engenharia Florestal) - Universidade Federal de Lavras, Lavras, 2004

FERREIRA, S. O. Estudo da forma do fuste de Eucalyptus grandis e Eucalyptus cloeziana. 132 p. Dissertação (Mestrado em Engenharia Florestal) - Universidade Federal de Lavras, Lavras, 1999.

FIGUEIREDO FILHO, A.; BORDERS, B. E.; HITCH, K. L. Taper equations for Pinus taeda plantations in southern Brazil. Forest Ecology and Management, Amsterdam, v. 83, n. 1-2, p. 36-46, 1996.

HRADETZKY, J. Analyse und interpretation statistisher abränger keiten (Biometrische Beiträge zu aktuellen forschungs projekten). Baden - Württemberg Mitteilungen der FVA, 1976. 146 p. (Abt. Biometric und Informatik, n. 21).

GRAYBILL, J. Theory and application of the linear model. Belmont: Duxbury, 1976.

IAPAR - Instituto Agronômico do Paraná. Cartas climáticas do Estado do Paraná: 1994. Londrina, 1994.

KOZAK, A.; MUNRO, D. P.; SMITH, J. H. G. Taper functions and their application in forest inventory. Forest Chronicle, Toronto, v. 45, n. 4, p. 278 - 283, 1969.

MAACK, R. Geografia física do Estado do Paraná. 2 ed. Rio de Janeiro: J. Olympio; Curitiba: Secretaria de Curitiba e do Esporte do Governo do Estado do Paraná. 1981. 450 p.

QUEIROZ, D.; MACHADO, S. A.; FIGUEIREDO FILHO, A.; ARCE, J. E.; KOEHLER, H. S. Avaliação e validação de funções de afilamento para Mimosa scabrella Bentham em povoamentos da região metropolitana de Curitiba. Floresta, Curitiba, v. 36, n. 2, maio/ago., 2006.

SCHÖEPPER, W. Autimatisierung des Massen, Sorten and Wertberechnung stenender Waaldbestande Schriftenreihe Bad. [S.I.]: Wurtt-Forstl., 1966. 